\title{
Control biológico de Rosellinia sp. causante de la muerte súbita en macadamia (Macadamia integrifolia) con aislados de Trichoderma spp.
}

\author{
Biological control of Rosellinia sp. causing of the sudden death of the macadamia \\ (Macadamia integrifolia) with isolates of Trichoderma spp.
}

\begin{abstract}
Andrés Dejesús Sanabria Velázquez ${ }^{1}$ y Cris thian J. Grabowski Ocampos ${ }^{2}$
${ }^{1}$ Instituto Paraguayo de Tecnología Agraria. Centro de Investigación Hernando Bertoni. Departamento Fitopatología. Caacupé, Paraguay. ${ }^{2}$ Universidad Nacional de Asunción. Facultad de Ciencias Agrarias. Área de Protección Vegetal. San Lorenzo, Paraguay.

* Autor para correspondencia (sanabria300@hotmail.com)

Recibido: 21/04/2015; Aceptado: 18/05/2016.

10.18004/investig.agrar.2016.dicie mbre.77-86
\end{abstract}

\section{RESUMEN}

Para evaluar la eficiencia del control biológico ejercido por ais lados nativos de Trichoderma spp. sobre Rosellinia sp. causante de la muerte de plantas de macadamia, se realizaron dos experimentos en el Laboratorio de Fitopatología de la FCA-UNA, San Lorenzo, Central y en el vivero San Joaquín, Caraguatay, Cordillera. Para las pruebas in vitro se obtuvieron 19 ais lados de Trichoderma spp. de parcelas productoras de macadamia del Paraguay y se enfrentaron en cultivo pareado con Rosellinia sp. para medir su capacidad antagónica, utilizando el Diseño Completamente al Azar (DCA) con cinco repeticiones. Los resultados obtenidos permitieron seleccionar a los aislados GS18 (Itapuá), GS13 (Central), GS10 (Cordillera) para su evaluación in vivo. Para el segundo experimento en condiciones in vivo se utilizó el DCA, los tratamientos consistieron en T1: Testigo (sólo el patógeno), T2: Mezcla de aislados de Trichoderma spp. y T3: Carbendazim al $0,1 \%$, con cinco repeticiones. Las plantas de macadamia de 12 meses fueron tratadas con los ais lados de Trichoderma spp.. Transcurridas 2 semanas se infestó el sustrato de todas las plantas con propágulos de Rosellinia spp.. Se aguardaron otras 2 semanas que permitieron el establecimiento del patógeno y se aplicó Carbendazim al 0,1\%. En las plantas testigo se constató $53 \%$ de incidencia de la enfermedad, en las tratadas con Trichoderma spp. $26 \%$, y en las plantas tratadas con Carbendazim se verificó $7 \%$. Se concluye que los aislados nativos Trichoderma spp. presentan potencial como agentes biocontroladores de Rosellinia sp. al reducir la incidencia de la enfermedad en condiciones in vivo.

Palabras clave: antagonismo, hongos, aislados nativos, agente biocontrolador, patógeno radicular.

\section{ABSTRACT}

To evaluate the biological control exercised by native isolates of Trichoderma spp. on Rosellinia sp., responsible for the death of macadamia nut plants, two experiments were carried out at the Phytopathology Lab of the FCA-UNA, San Lorenzo, main campus and at the San Joaquín nursery, Caraguatay, Cordillera. For in vitro tests, 19 isolates of Trichoderma spp. were obtained from several macadamia growing plots in Paraguay. They were faced in matched crops with Rosellinia sp. to measure their antagonistic capacity, using a completely randomized design with five repetitions. The results allowed selecting the isolates GS18 (Itapua), GS13 (Central), GS10 (Cordillera) for their in vivo evaluation. For the second experiment, a completely randomized design was used. The treatments consisted on T1: Control (the pathogen only), T2: Trichoderma spp isolates mix. and T3: $1 \%$ Carbendazim having each one five repetitions. 12 months old Macadamia plants were treated with the Trichoderma spp isolates mix. After 2 weeks, the substratum of all plants was infested with Rosellinia sp. propagules, two more weeks were allowed to assure the establishment of the pathogen and then Carbendazim with $0,1 \%$ dose, was applied. In the control plants, it was observed a $53 \%$ incidence of disease, a $26 \%$ in the plants treated with the Trichoderma spp. mix and $7 \%$ in plants treated with Carbendazim. It is concluded that native isolates of Trichoderma spp. have a potential as Rosellinia sp .bio-control agents since they reduce the incidence of the disease in in vivo conditions.

Key words: antagonism, fungi, native isolates, biocontrol agent, roots pathogen. 


\section{INTRODUCCIÓN}

La macadamia (Macadamia integrifolia Maiden \& Betche) es un cultivo comercial de importancia creciente debido a sus refinadas nueces, las cuales son muy apreciadas en toda Europa, Japón y Estados Unidos. Las nueces son utilizadas en el sector de "snacks," confitería, heladería, industria del chocolate, repostería, su aceite es utilizado en productos dermatológicos y cosméticos (Armadans 2007). Al igual que otros cultivos, la macadamia es susceptible al ataque de diversos hongos causantes de daños y perdidas en la producción. Los patógenos fúngicos Botryodiplodia sp. y Rosellinia pepo, agente causal de la llaga estrellada o muerte súbita, son los de mayor importancia al causar pérdidas en el rendimiento del cultivo (Villegas 2005).

El hongo Rosellinia sp. es un parásito facultativo, su distribución es cosmopolita, reportándose alrededor de todo el mundo, además posee un amplio rango de hospederos causando pérdidas económicas en varios cultivos y árboles frutales. En Argentina se han reportado varias especies del género Rosellinia spp., entre ellas $R$. necatrix, $R$. pepo, $R$. bunodes y $R$. paraguayensis (Sir et al. 2012). En Paraguay fue reportado recientemente como agente causal de la muerte de plantas de macadamia en su etapa productiva (Grabowski et al. 2014), pudiendo llegar a presentar una incidencia entre el 5 y el $70 \%$, comprometiendo anualmente áreas nuevas de siembra (Villegas et al. 2006).

El hongo se establece inicialmente en las raíces secundarias, penetrando la raíz por medio de estructuras especializadas llamadas apresorios, razón por lo cual la invasión del sistema radical es lenta, pero progresiva. Una vez que se establece en los tejidos de la base del árbol se presenta un debilitamiento, con posterior amarillamiento del follaje y una ausencia de emisión de brotes nuevos; después de algunas semanas el árbol muere. Las hojas se tornan de un color rojizo y quedan adheridas por varias semanas (Mendoza 2000, Grabowski et al. 2014). Los factores que favorecen el desarrollo del patógeno son la presencia de árboles viejos de sombrío con sus raíces en proceso de descomposición, los altos contenidos de materia orgánica en la superficie del suelo y la frecuencia de las lluvias, observándose una incidencia casi insignificante en donde la frecuencia de las lluvias es baja y existe escasa acumulación de humus y materia orgánica (Mendoza et al. 2002).
Teniendo en cuenta la falta de productos fungicidas indicados para el manejo de la enfermedad debido a la baja eficiencia que estos presentan en el suelo (Sharma y Gupta 1985); el control biológico es una propuesta prometedora, ya que esta técnica está directamente involucrada a la selección y reintroducción de un antagonista, que pueda establecerse en el mismo nicho ecológico del patógeno y pueda proteger el sistema radicular de la planta de macadamia, reduciendo la intensidad de la enfermedad.

Varios autores como Aránzazu (1996), Mendoza et al. (2002), Cazorla et al. (2006) mencionan que el uso de antagonistas, principalmente de los géneros Trichoderma sp. y Pseudomonas sp., es el método más prometedor para el combate de Rosellinia spp. debido a los resultados positivos obtenidos en ensayos utilizando estos mismos microorganismos para el control del fitopatógeno. El éxito de Trichoderma spp.para controlar patógenos de suelo se debe a que estos antagonistas son ais lados de suelos con condiciones ecológicas similares a los que son reintroducidos, asegurando su capacidad de competencia y expresión de más de un mecanismo antagónico, lo cual ya había sido mencionado por Harman et al. (2004) y Harman (2006).

El objetivo del presente trabajo fue evaluar la eficiencia del control biológico ejercido por los aislados nativos de Trichoderma spp. sobre el fitopatógeno radicular de la macadamia Rosellinia sp., en condiciones in vitro e in vivo.

\section{MATERIALES Y MÉTODOS}

Los experimentos se realizaron en el Laboratorio de Fitopatología del Área de Protección Vegetal de la Facultad de Ciencias Agrarias (FCA) de la Universidad Nacional de Asunción (UNA), San Lorenzo, Central y en el vivero de la finca productora de macadamia San Joaquín ubicada en la ciudad de Caraguatay, Cordillera. El periodo de experimentación estuvo comprendido entre los meses de octubre de 2013 y febrero de 2014.

Los aislados nativos de Trichoderma spp. fueron obtenidos a partir de muestras de suelos provenientes de diferentes fincas de producción de macadamia del Paraguay (Tabla 1). El aislamiento de Trichoderma spp. se realizó mediante una adaptación del método de dilución del suelo citado por Fernández (1993). Este método consistió en diluir $3 \mathrm{~g}$ de suelo en $100 \mathrm{cc}$ de agua destilada estéril y agitar la suspensión. Posteriormente 
bajo la cámara de flujo laminar se retiró y colocó 1 cc de la suspensión en una placa de Petri esterilizada. Luego, se adicionó el medio de cultivo PDA (Papa- Dextrosa- Agar) + Oxitetraciclina, y se agitó suavemente. Las placas fueron incubadas a $28^{\circ} \mathrm{C}$ durante 5 días en la incubadora.
Los cultivos puros de Trichoderma spp. fueron codificados con el termino GS (nomenclatura arbitraria) y un número, de manera a diferenciarlos de otros aislados nativos de Trichoderma spp.,

Tabla 1. Muestras de suelo georreferenciadas para la obtención de aislados de Trichoderma spp. FCA-UNA. San Lorenzo, Paraguay. 2014.

\begin{tabular}{cccc}
\hline $\begin{array}{c}\text { Código de la } \\
\text { muestra }\end{array}$ & Aislados de Trichoderma spp. & Localidad & Coordenadas \\
\hline 1 & GS1, GS2 & Paraguarí & 21 J 475359 UTM 7174680 \\
2 & GS3 & Cordillera & 21 J 491517 UTM 7174368 \\
4 & GS4, GS5 & Cordillera & 21 J 500410 UTM 7183024 \\
5 & GS6, GS7 & Central & 21 J 0464904 UTM 7189080 \\
6 & GS8, GS9 & Cordillera & 21 J0467008 UTM 7212897 \\
$6 \mathrm{R}$ & GS10, GS11 & Cordillera & 21 J0467008 UTM 7212897 \\
6 Saico & GS12 & Cordillera & 21 J0467008 UTM 7212897 \\
7 & GS13, GS14 & Central & 21 J0440920 UTM 7190631 \\
$8 \mathrm{M}$ & GS15 & Central & 21 J0440920 UTM 7190631 \\
10 & GS16, GS17 & Itapúa & 21 J0606252 UTM 7005321 \\
$10 B$ & GS18, GS19 & Itapúa & 21 J0606252 UTM 7005321 \\
\hline
\end{tabular}

El hongo Rosellinia sp. fue aislado de plantas con síntomas de muerte súbita o problemas radiculares de las parcelas de producción de macadamia del Paraguay. Las raíces colectadas de plantas de macadamia se lavaron con agua corriente y fueron cortadas en trozos de 1 a $2 \mathrm{~cm}$, después se desinfestaron mediante una solución con alcohol al $70 \%$ y otra de hipoclorito de sodio al $2 \%$, seguidamente se realizó un triple enjuague en agua destilada estéril, se dejó secar el tejido vegetal y se transfirió a placas de Petri con medio de cultivo EMA (Extracto de Malta-Agar) bajo cámara de flujo laminar. Posteriormente, se dejaron incubar a $28^{\circ} \mathrm{C}$ durante 8 días (Villegas et al. 2006). Para el reconocimiento de Rosellinia sp. se observaron las colonias mediante el estereoscopio y el microscopio óptico identificando las estructuras típicas del hongo, concordando con Villegas et al. (2006) y Mendoza (2000) quienes describen al patógeno como un hongo de crecimiento lento en medio de cultivo, con micelio de aspecto algodonoso el cual a medida que envejece se torna de color gris ahumado a café. También se observaron los hinchamientos piriformes característicos del género Rosellinia mencionados por los mismos autores.

\section{Antagonismo in vitro de Trichoderma spp. ante Rosellinia sp.}

Se utilizó un diseño completamente al azar (DCA) con 20 tratamientos siendo 19 los aislados de Trichoderma spp., enfrentados cada uno con Rosellinia sp., y un testigo consistente en un disco con medio de cultivo y micelio de Rosellinia sp.. Cada tratamiento contó con cinco repeticiones, resultando 100 unidades experimentales, las cuales consistieron en una placa de Petri cada una.

Para seleccionar los aislados de Trichoderma spp. con capacidad antagónica ante Rosellinia sp. se empleó la técnica de cultivo pareado mencionada por Bell et al. (1982), según la cual se obtuvieron discos de $5 \mathrm{~mm}$ de diámetro del crecimiento micelial de Trichoderma spp. y del fitopatógeno Rosellinia sp., los cuales se transfirieron a placas de Petri de $90 \mathrm{~mm}$ conteniendo medio de cultivo PDA y se llevaron a incubación a $28^{\circ} \mathrm{C}$ hasta que el tratamiento testigo llenó la placa de Petri.

Se evaluó la actividad antagónica de los aislados de Trichoderma spp. midiendo la velocidad de crecimiento micelial promedio (mm/día) y la sobreposición del micelio de Trichoderma spp. y el fitopatógeno Rosellinia 
sp., para ello se utilizó la escala propuesta por Bell et al. (1982), detallada en la Tabla 2.

También se calculó el porcentaje de inhibición de crecimiento del patógeno mediante la siguiente fórmula adaptada de Orrego et al. (2013):

Donde:

$$
\mathrm{I}=100-\left[\left(\mathrm{LMP}_{\mathrm{CP}} / \mathrm{LMP}_{\mathrm{CI}}\right)\right]^{*} 100
$$

I: Inhibición del crecimiento de Rosellinia sp. (\%)

LMP ${ }_{\mathrm{CP}}$ : Longitud del crecimiento de Rosellinia sp. en cultivo pareado $(\mathrm{mm})$

LMP $_{\mathrm{CI}}$ : Longitud del crecimiento de Rosellinia sp. en cultivo individual $(\mathrm{mm})$

Tabla 2. Escala propuesta por Bell et al. (1982) para la evaluación y clasificación del antagonismo. FCA-UNA. San Lorenzo, Paraguay. 2014.

\begin{tabular}{cl}
\hline Índice & \multicolumn{1}{c}{ Descripción } \\
\hline 1 & $\begin{array}{l}\text { Trichoderma spp. sobrecrece completamente } \\
\text { al patógeno y cubre totalmente la superficie } \\
\text { del medio }\end{array}$ \\
2 & $\begin{array}{l}\text { Trichoderma spp. sobrecrece las dos terceras } \\
\text { partes de la superficie del medio }\end{array}$ \\
3 & $\begin{array}{l}\text { Trichoderma spp. y el patógeno colonizan } \\
\text { cada uno aproximadamente la mitad de la }\end{array}$ \\
& $\begin{array}{l}\text { superficie y ningún organismo parece } \\
\text { dominar al otro } \\
\text { El patógeno coloniza las dos terceras partes } \\
\text { de la superficie del medio y parece resistir a } \\
\text { la invasión por Trichoderma spp. } \\
\text { El patógeno sobrecrece completamente a } \\
\text { Trichoderma spp. }\end{array}$ \\
\hline
\end{tabular}

\section{Eficiencia de Trichoderma spp. en el control de Rosellinia sp. en condiciones in vivo}

Se utilizó el diseño completamente al azar (DCA), con tres tratamientos y cinco repeticiones como se observa en la Tabla 3. Se consideró como unidad experimental tres macetas con una planta de macadamia cada una, totalizando 45 plantas.

El tratamiento $\mathrm{T} 1$ (testigo) consistió en plantas con sustrato infestado sólo con el fitopátogeno Rosellinia sp. Por otro lado, en el tratamiento T2 el sustrato de las plantas fue tratado con una mezcla de tres aislados de Trichoderma spp. más eficientes en las pruebas in vitro. En el tratamiento T3 fue aplicado el fungicida Carbendazim al $0,1 \%$ de acción sistémica, compuesto por 2-metoxicarbamoil-bencimidazol $\quad(50 \quad \mathrm{~g}), \quad$ agentes dispersantes y solventes c.p.s $\left(100 \mathrm{~cm}^{3}\right)$, formulado como suspensión concentrada (SC).

Durante los experimentos fueron utilizadas plantas injertadas de macadamia de la variedad HAES 344 de 12 meses de edad. Las dimensiones de las macetas de las plantas fueron $15 \mathrm{~cm}$ de diámetro y $45 \mathrm{~cm}$ de alto, conteniendo como sustrato arena gorda y materia orgánica en proporción 2:1. Las plantas fueron ubicadas bajo malla media sombra $(50 \%)$ registrándose temperaturas de $30 \pm$ $5^{\circ} \mathrm{C}$. El riego se realizó periódicamente de manera a mantener la humedad del sustrato a capacidad de campo.

Para la incorporación de los propágulos de Trichoderma spp. al sustrato de las macetas con plantas de macadamia, se procedió a mezclar y homogeneizar en un solo recipiente el arroz colonizado por los tres aislados nativos seleccionados mediante las pruebas de antagonismo in vitro y se dispersó $50 \mathrm{~g}$ en cada maceta a un nivel uniforme de profundidad (López-Herrera et al. 1999).

Al cabo de dos semanas posteriores al establecimiento del agente de control biológico, se infestaron las macetas con propágulos de Rosellinia sp. incorporando $250 \mathrm{~g}$ de arroz colonizados por el patógeno a un nivel uniforme de profundidad regando posteriormente con agua, según la metodología mencionada por López-Herrera et al. (2003) y Grabowski et al. (2014).

La aplicación del fungicida Carbendazim se realizó 15 días posteriores a la infestación del sustrato con el patógeno Rosellinia sp.. El equipo empleado para realizar la aplicación fue el pulverizador tipo mochila de $20 \mathrm{~L}$ con pico del tipo cono hueco.

La evaluación se realizó determinando la incidencia de la enfermedad, es decir el número de plantas muertas en cada tratamiento, mediante observaciones semanales a partir de las cuatro semanas posteriores a la inoculación del patógeno, tiempo en el cual se observaron los primeros signos del patógeno.

Los datos obtenidos, en ambos experimentos, fueron sometidos al análisis de Varianza (ANAVA), y al encontrarse diferencias significativas entre los tratamientos se aplicó el Test de Tukey al 5\% de probabilidad de error. Los datos de incidencia en plantas de macadamia fueron transformados mediante $\lfloor(\sqrt{Y})+1\rfloor_{\mathrm{y}}$ luego se realizó el ANAVA. Para los 
análisis estadísticos se utilizó el paquete estadístico InfoStat ${ }^{\circledR}$ (Di Rienzo et al. 2008).

Tabla 3. Tratamientos aplicados en plantas de macadamia en vivero. FCA-UNA. San Lorenzo, Paraguay. 2014.

\begin{tabular}{cl}
\hline Tratamiento & \multicolumn{1}{c}{ Descripción } \\
\hline T1 & Testigo \\
T2 & Mezcla de aislados seleccionados de \\
& Trichoderma spp. \\
T3 & Carbendazim $(0,1 \%)$ \\
\hline
\end{tabular}

\section{RESULTADOS Y DISCUSIÓN}

Antagonismo in vitro de Trichoderma spp. ante Rosellinia sp.

Sobreposición micelial de Trichoderma spp. sobre Rosellinia sp.; en la Tabla 4 se observa el crecimiento micelial lineal medido en mm/día para los 19 aislados de Trichoderma spp. a las 72 h de incubación.

Se hallaron diferencias significativas entre los aislados nativos de Trichoderma spp. en cuanto a su velocidad de crecimiento lineal, registrándose valores de17,85 mm/día hasta $30,37 \mathrm{~mm} /$ día.

Los valores de crecimiento micelial lineal de los aislados estudiados en este experimento fueron mayores a los verificados en trabajos anteriores de control de fitopatógenos del suelo con aislados de Trichoderma spp. como los de Franco y Orrego (2013) quienes registraron valores máximos de 20,25 mm/día, también Garcete y Orrego (2011) obtuvieron valores de 17,00 mm/día ambos para cultivo pareado con Macrophomina phaseolina, mientras que Manzur (2011) observó valores máximos de $18,10 \mathrm{~mm} /$ día de crecimiento micelial promedio contra el hongo del suelo Sclerotium rolfsii. Por su parte Valencia y Castro (2004) registraron velocidades de crecimiento micelial de $22,5 \mathrm{~mm} /$ día en condiciones de luz al seleccionar aislados de Trichoderma spp. para el control de Rosellinia bunodes.

En cuanto a los grados de la escala de Bell et al. (1982) también se verificaron diferentes valores para los aislados de Trichoderma spp.. Para los aislados GS18, GS13, GS1, GS2, GS10, GS3, GS4 y GS15 se registraron valores igual a 1, lo que significa que sobrecrecieron completamente a Rosellinia sp. y cubrieron totalmente la superficie del medio. Por otra parte, para los aislados
GS8, GS11, GS5, GS19, GS12, GS6, GS7, GS14 y GS9 se verificaron valores igual a 2 , lo que equivale a que los aislados de Trichoderma spp. sobrecrecieron las dos terceras partes de la superficie del medio. Los aislados GS16, GS17 y el patógeno Rosellinia sp. colonizaron cada uno aproximadamente la mitad de la superficie y ningún organismo pareció dominar al otro obteniendo el valor 3 de la escala.

Tabla 4. Velocidad y escala de crecimiento lineal de aislados de Trichoderma spp. en las pruebas de antagonismo in vitro con Rosellinia sp. FCAUNA. San Lorenzo, Paraguay. 2014.

\begin{tabular}{|c|c|c|}
\hline Aislado & Velocidad (mm/día)* & Escala de Bell** \\
\hline GS18 & $30,37 \quad \mathrm{a}$ & 1 \\
\hline GS13 & 29,64 a & 1 \\
\hline GS1 & 29,28 a & 1 \\
\hline GS2 & 28,73 a & 1 \\
\hline GS10 & 28,56 a & 1 \\
\hline GS3 & $28,51 \quad \mathrm{a}$ & 1 \\
\hline GS4 & 27,56 a & 1 \\
\hline GS15 & 27,30 a & 1 \\
\hline GS8 & 26,75 a & 2 \\
\hline GS11 & 26,64 a b & 2 \\
\hline GS5 & 25,82 a b c & 2 \\
\hline GS19 & 25,24 a b c & 2 \\
\hline GS12 & 25,16 a b c & 2 \\
\hline GS6 & 24,86 a b c & 2 \\
\hline GS7 & 24,77 a b c & 2 \\
\hline GS14 & 24,34 a b c & 2 \\
\hline GS9 & 24,20 a b c & 2 \\
\hline GS16 & $18,29 \quad b \quad c$ & 3 \\
\hline GS17 & 17,85 & 3 \\
\hline
\end{tabular}

(*)Medias seguidas por la misma letra no difieren entre sí por el test de Tukey al 5\%

(**)Escala adaptada de Bell et al. (1982)

Según los mismos autores la técnica de selección in vitro basada en esta escala, puede llegar a ser solo un acercamiento simplista a un sector pequeño de los sistemas biológicos implicados en el control de enfermedades. Sin embargo, provee información útil acerca de la variabilidad de los distintos aislamientos de Trichoderma en cuanto a su capacidad de antagonizar a los patógenos. 
Los trabajos mencionados anteriormente coinciden en que existe una variabilidad entre los aislados nativos de Trichoderma spp. de diferentes zonas agroecológicas en cuanto a su capacidad de sobrecrecer al patógeno y cubrir la superficie del medio, lo cual ya había sido destacado por Papavizas (1985).

La variabilidad de estos hongos agentes de control biológico y la expresión de sus diferentes mecanismos de antagonismo, se deben principalmente a la adaptación de los diferentes ais lados a sus respectivos ecosistemas y a la interacción entre los componentes patógeno-plantaantagonista, debido a lo cual no todos los aislados seleccionados tienen la misma capacidad de expresar sus diferentes mecanismos de antagonismo, en este caso la competencia por espacio (Bautista et al. 2008, Infante et al. 2009, Cordero y Maniscalco 2010). Por esta razón a pesar de que todos los aislados fueron obtenidos de parcelas de producción de macadamia, estos presentaron diferentes grados de sobreposición.

Inhibición de crecimiento in vitro de Rosellinia sp.; en la Tabla 5 son detallados los porcentajes de inhibición de crecimiento de Rosellinia sp. ejercido por los aislados de Trichoderma spp. en cultivo pareado.

Se verificaron diferencias significativas entre los aislados de Trichoderma spp. en cuanto a los porcentajes de inhibición de crecimiento de Rosellinia sp. en los experimentos de antagonismo in vitro, registrándose porcentajes de inhibición de crecimiento de $42,80 \%$ hasta $80,20 \%$. Estos valores son superiores a los registrados por Ruano-Rosa et al. (2010) quienes observaron porcentajes de inhibición de crecimiento de Rosellinia sp. de 39,80 hasta $14,90 \%$.

Lo registrado en este experimento coincide con Esquivel et al. (1992) quienes observaron in vitro que todos los aislados del antagonis ta fueron excelentes competidores al patógeno, debido a su rápida tasa de desarrollo. El crecimiento de las colonias de Rosellinia en placas de Petri fue inhibido por el crecimiento de la colonia de Trichoderma spp. que creció y esporuló sobre ella. Por su parte, Ruano-Rosa et al. (2003) también observaron que todos los ais lados de Trichoderma spp., excepto el aislado CH-252, limitaron el crecimiento del patógeno Rosellinia necatrix, observándose poca variación en su efecto para cada ais lado del antagonista sobre el patógeno.

Estos resultados demuestran que los aislados de Trichoderma spp. presentan potencial como controladores biológicos de Rosellinia sp. al inhibir el crecimiento micelial del patógeno coincidiendo con otros autores como Mendoza et al. (2002); López-Herrera et al. (2003); Ruano-Rosa et al. (2003); Valencia y Castro (2004); Ruano-Rosa et al. (2010), quienes también seleccionaron aislados nativos de Trichoderma para el control de patógenos de suelo.

Tabla 5. Inhibición de crecimiento in vitro de Rosellinia sp. por efecto del enfrentamiento con diferentes aislados de Trichoderma spp. en cultivo pareado. FCA-UNA. San Lorenzo. Paraguay. 2014.

\begin{tabular}{|c|c|c|}
\hline Aislado & $\begin{array}{l}\text { Inhibición de ci } \\
\text { Rosellinia }\end{array}$ & $\begin{array}{l}\text { cimiento de } \\
(\%)^{*}\end{array}$ \\
\hline GS13 & 80,20 & $\mathrm{a}$ \\
\hline GS18 & 78,40 & $\mathrm{a}$ \\
\hline GS10 & 75,40 & $\mathrm{a}$ \\
\hline GS8 & 72,40 & $\mathrm{a}$ \\
\hline GS4 & 71,80 & $\mathrm{a}$ \\
\hline GS1 & 70,80 & $\mathrm{a}$ \\
\hline GS11 & 69,60 & $\mathrm{a}$ \\
\hline GS6 & 68,20 & $\mathrm{a}$ \\
\hline GS19 & 68,00 & $\mathrm{a}$ \\
\hline GS2 & 67,00 & $\mathrm{a}$ \\
\hline GS15 & 66,80 & $\mathrm{a}$ \\
\hline GS9 & 66,40 & $\mathrm{a}$ \\
\hline GS14 & 66,00 & $\mathrm{a}$ \\
\hline GS12 & 65,20 & $\mathrm{a}$ \\
\hline GS7 & 64,20 & $\mathrm{a}$ \\
\hline GS4 & 63,80 & $\mathrm{a}$ \\
\hline GS3 & 63,60 & $\mathrm{a}$ \\
\hline GS17 & 42,80 & b \\
\hline GS16 & 42,80 & $\mathrm{~b}$ \\
\hline Testigo & 0,00 & $\mathrm{c}$ \\
\hline
\end{tabular}

(*)Medias seguidas por la misma letra no difieren entre sí por el test de Tukey al 5\%

Fueron seleccionados los aislados de Trichoderma spp. GS13, GS18 y GS10, debido a la eficiencia que exhibieron en el control de Rosellinia sp. en las pruebas de antagonismo in vitro. También López-Herrera et al. (1999) seleccionaron diferentes aislados de Trichoderma spp. (T6: Trichoderma aureoviridae, T9 y T10: Trichoderma longibrachiatum, TB1.2: Trichoderma harzianum) teniendo en cuenta su capacidad para antagonizar al patógeno Rosellinia necatrix en condiciones in vitro, verificándose su eficiencia al reducir 
un $37 \%$ el porcentaje de aislamiento del patógeno de raíces de plantas de aguacate durante los experimentos in vivo.

\section{Eficiencia de Trichoderma spp. en el control de Rosellinia sp. en condiciones in vivo}

En la Figura 1 se detalla la incidencia del ataque de Rosellinia sp. sobre plantas de macadamia en cada tratamiento durante las pruebas in vivo.

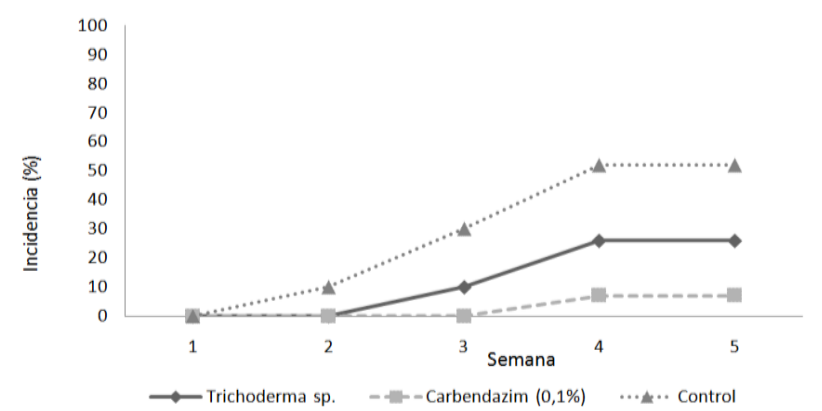

Figura 1. Porcentaje de incidencia del ataque de Rosellinia sp. sobre plantas de macadamia. FCA-UNA. San Lorenzo, Paraguay. 2014.

En el tratamiento testigo se observó los primeros signos y síntomas en las plantas a los 30 días de la inoculación con el patógeno, progresando hasta alcanzar una incidencia de $53 \%$, diferenciándose estadísticamente del tratamiento Carbendazim en el cual la incidencia fue del $7 \%$. Para la mezcla de aislados de Trichoderma spp. fue observada una incidencia de $26 \%$, no presentando diferencias significativas con ninguno de los tratamientos.

Estos resultados son similares a los obtenidos por Valencia y Castro (2004) quienes observaron una incidencia de Rosellinia bunodes en raíces de plantines de café del 20 al 28\% tratadas con aislados de Trichoderma spp., mientras que el tratamiento testigo presentó un $64 \%$ de plantines enfermos al cabo de 30 días de la inoculación con el patógeno. También Oliveira et al. (2008) observaron síntomas y signos del patógeno 32 días posteriores a la inoculación.

Ruano-Rosa et al. (2003) registró que los aislados de Trichoderma spp. que ejercieron un mejor control sobre la enfermedad causada por Rosellinia necatrix fueron $\mathrm{CH}$ 255 y $\mathrm{CH} 316$, observándose para ambos tratamientos todas las plantas sanas, el CH 303 presentó un 30\% de plantas muertas y los aislados $\mathrm{CH} 218, \mathrm{CH} 256, \mathrm{CH} 300$, CH304, CH314 más de 30\% de plantas muertas, no diferenciándose significativamente del testigo inoculado con el patógeno, concluyendo también que sería conveniente la utilización de combinaciones de aislados para potenciar su efecto antagonista sobre el patógeno.

Los microorganismos antagonistas como Trichoderma spp. son capaces de ejercer un control preventivo al colonizar las raíces de las plantas las cuales son su hábitat natural, proliferando en simbios is con la planta, regulando las poblaciones de patógenos e induciendo resistencia en las mismas (Infante et al. 2009). Esto se verificó al examinar las raíces de las plantas de macadamia tratadas con la mezcla de aislados de Trichoderma spp. (Figura 2).

Cabe destacar que el control biológico permite regular las poblaciones de microorganismos patógenos, pero no eliminarlos por completo (Aguilar 1980), este hecho es avalado por los resultados obtenidos en esta investigación donde en las plantas tratadas con ais lados de Trichoderma spp. se registró un $26 \%$ de incidencia comparado a las tratadas con Carbendazim, en las cuales se observó un $7 \%$ de plantas enfermas. Lopéz-Herrera et al. (2003) mencionan que el fungicida Carbendazim presentó cierta eficiencia en el control de Rosellinia sp. durante ensayos con plantas de aguacate, sin embargo, la baja persistencia del fungicida en el suelo se tradujo en la aparición de síntomas de la enfermedad pasados 30 días de una segunda inoculación con el patógeno.

Para los aislados de Trichoderma spp. GS13, GS18 y GS10 fue observado un efecto antagónico frente a Rosellinia sp. tanto durante las pruebas in vitro como posteriormente en las pruebas in vivo. Estos aislados obtenidos de parcelas productoras de macadamia fueron eficientes al controlar a Rosellinia sp. durante las pruebas in vivo debido a su gran velocidad de crecimiento y su alta capacidad para colonizar el sustrato, permitiendo comprobar el potencial como agentes de control biológico que presentaron durante las pruebas in vitro. 

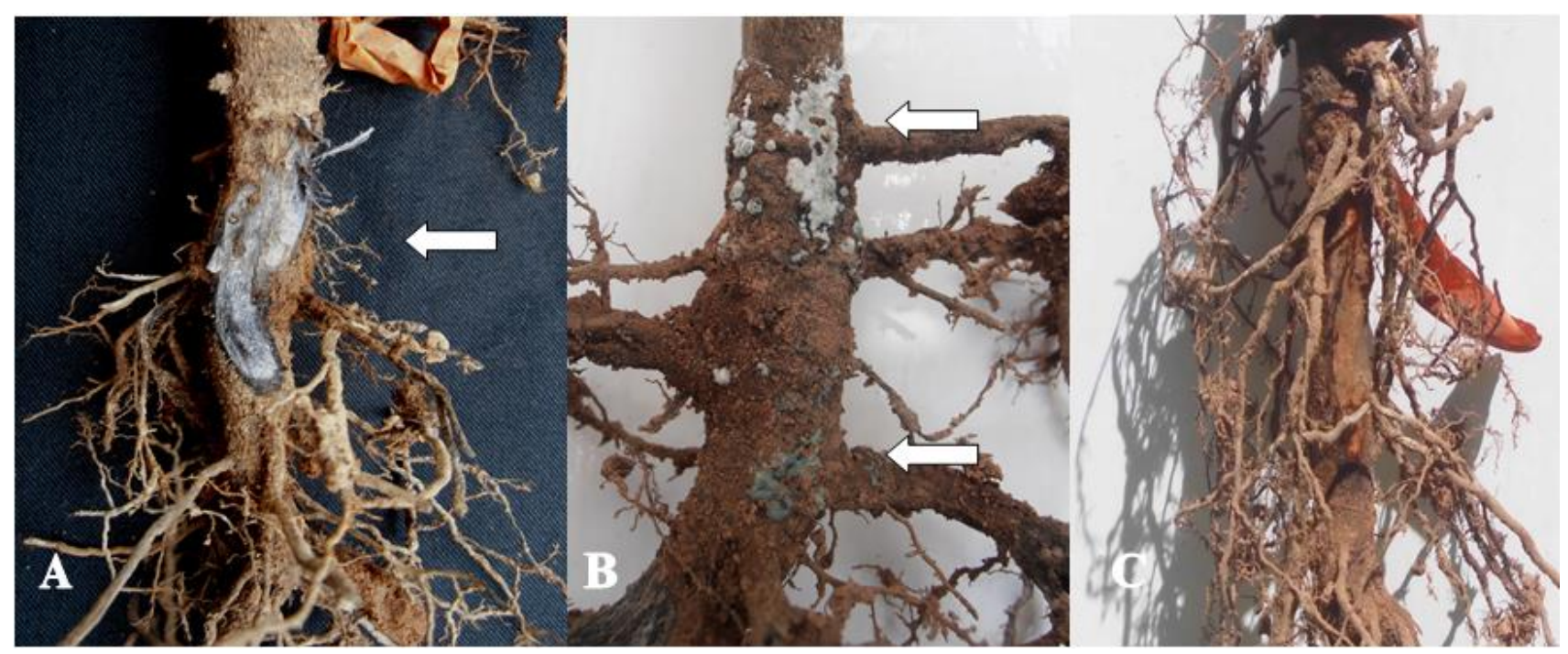

Figura 2. Sistema radicular de plantas de macadamia. A) Micelio del patógeno Rosellinia sp. (flecha) creciendo bajo la corteza del sistema radicular en el tratamiento testigo. B) Colonias de color blanco y verde correspondientes a Trichoderma spp. (flechas) en plantas tratadas con la mezcla de aislados. C) Ausencia de crecimiento fúngico en raíces tratadas con Carbendazim. FCA-UNA. San Lorenzo, Paraguay. 2014.

\section{CONCLUSIONES}

En condiciones in vitro los aislados nativos de Trichoderma spp. obtenidos de diferentes parcelas productoras de macadamia presentan diferencias en cuanto a la velocidad de crecimiento, el grado de sobreposición micelial y su capacidad de inhibición de crecimiento in vitro de Rosellinia sp., siendo seleccionados los aislados de Trichoderma spp. GS13, GS18, GS10, GS8, GS4, GS1, GS11, GS6, GS19, GS2, GS15, GS9, GS14, GS12, GS7, GS4 y GS3 por presentar potencial antagónico ante Rosellinia sp..

En condiciones in vivo la mezcla de aislados nativos de Trichoderma spp. seleccionados mediante pruebas in vitro (GS13, GS18, GS10) reduce la incidencia de la enfermedad causada por Rosellinia sp. en plantas de macadamia.

\section{REFERENCIAS BIBLIOGRAFICAS}

Aguilar, P. 1980. Apuntes sobre el control biológico y el control integrado de las plagas agrícolas en el Perú (en línea). Consultado 28 jul 2012. Disponible en http://www.revperuentomol.com.pe /publicaciones/ vol23/CONTROL-BIOLOGICO-Y-CONTROL-INTE GRADO-EN-EL-PERU83.p df

Aránzazu, HF. 1996. Comportamiento de la llaga estrellada Rosellinia pepo Pat. sobre raíces de cacao (en línea). Fitopatología Colombiana 20(1):7-10. Consultado 28 jul 2013. Disponible en http://agris .fao.org/agris-search/s earch.do?recordID=C O1999 002871

Armadans, A. 2007. Cultivo de macadamia en el Paraguay. San Lorenzo, Paraguay, FCA-UNA. 63 p.

Bautista, CJ; García, R; Pérez, J; Zavaleta, E; Montes, R; Ferrera, R. 2008. Inducción de supresividad a fitopatógenos del suelo; un enfoque holístico al control biológico (en línea). Interciencia. 33(2):96102. Consultado 15 ene 2014. Disponible en http://www.scielo.org.ve /pdf/inci/v33n2/art05.pdf

Bell, DK; Wells, HD; Markham, CR. 1982. In vitro antagonism of Trichoderma species against six fungal pathogens (en línea). Phytopathology 72(4):379382.Consultado 28 dic 2012. Disponible en http://www.cabdirect.org/abstracts/19821384099.html ;jses sionid=12E7A 977CD30D71D33BCF5D82A243B 62

Cazorla, F; Duckett, S; Bergström, E; Noreen, S; Odijk, R; Lugtenberg, B; Thomas-Oates, J; Bloemberg, G. 2006. Biocontrol of avocado Dematophora root rot by antagonistic Pseudomonas fluorescens PCL1606 correlates with the production of 2-hexyl 5-propyl resorcinol (en línea). Plant Disease 19(4):418-428. 
Consultado 3 feb 2013. Disponible en http://apsjournals .apsnet.org /doi/pdfplus/10.1094/MP MI-19-0418

Cordero, MR; Maniscalco, DP. 2010. Diversidad de Trichoderma spp. en plantaciones de Theobroma cacao 1. del estado Carabobo, Venezuela, y su capacidad biocontroladora sobre Crinipellis pernicosa (Stahel) Singer (en línea). Interciencia 35(10):777783. Consultado 3 feb. 2013. Disponible en http://www.interciencia.org/v35_10/777.pdf

Di Rienzo, JA; Casanoves, F; Balzarini, MG; Gonzalez, L; Tablada, M; Robledo, CW. 2008. InfoStat, versión 2008. Argentina, Grupo InfoStat, FCA, Universidad Nacional de Córdoba, Argentina. 336p.

Esquivel, VH; Leguizamón, JE; Arbeláez, G. 1992. Búsqueda y evaluación de antagonistas a Rosellinia bunodes, agente causante de la llaga negra del cafeto (en línea). Cenicafé 43(2):33-42. Consultado 15 dic 2013. Disponible en http://orton.catie.ac.cr/cgibin /wxis .exe/?Is is Script=CAFE.xis \&method=post\&form at

Fernández, M. 1993. Manual para laboratorio de Fitopatología. San José, Costa Rica, IICA. 289 p.

Franco, B; Orrego, A. 2013. Compatibilidad in vitro de aislados nativos de Trichoderma spp. con fungicidas para el tratamiento de semillas (en línea). Investigación agraria 15(1): 15-22. Consultado 15 ene $2014 . \quad$ Disponible en http://www.agr.una.py/revista/index.php/ria/ article/view/2/2

Garcete, JM; Orrego, A. 2011. Efecto de aislados nativos de Trichoderma spp. en la incidencia de Macrophomina phaseolina (Tassi) Goid en sésamo (Sesamum indicum L.) (en línea). Investigación agraria 13(2):87-93. Consultado 15 ene 2014. Disponible en http://www.agr.una.py/revista/ index.php /ria/article/view/220/213

Grabowski, C; Sanabria, A; Armadans, A. 2014. Caracterización e identificación del organismo causal de la muerte súbita de la macadamia (Macadamia integrifolia) en Paraguay. In III Congreso Nacional de Ciencias Agrarias. (3, 2014, San Lorenzo, Paraguay). Paraguay, FCA. 496 p.
Harman, GE; Howell, CR; Viterbo, A; Chet, L; Lorito, M. 2004. Trichoderma species opportunistic, avirulent plant symbionts (en línea). Nature Reviews 2(1):4356. Consultado 03 ene 2014. Disponible en http://www.ncbi.nlm.nih.gov/pubmed /15035008

Harman, GE. 2006. Overview of mechanisms and uses of Trichoderma spp. symposium the nature and application of biocontrol microbes II: Trichoderma spp. (en línea). The American Phytopathological Society. Cornell University, 96(2): 190-194. Consultado 23 ene 2014. Disponible en http://www.hort.cornell.edu/department/faculty/harma n/pubs/06APSsymp.pdf

Infante, D; Martínez, B; González, N; Reyes, Y. 2009. Mecanismos de acción de Trichoderma frente a hongos fitopatógenos (en línea). Revista de Protección Vegetal 24(1): 14-21. Consultado 23 ene 2013. Disponible en http://scielo.sld.cu/scielo.php?pid=S1 010- $27522009000100002 \&$ \&cript $=$ sci_arttext

López-Herrera, CJ; Pérez- Jiménez, RM; Llobel, A; Vazquez, EM; Zea-Bonilla, T. 1999. Estudios in vivo de Trichoderma como agente de biocontrol contra Phytophthora cinnamoni y Rosellinia necatrix en Aguacate (en línea). Revista Chapingo Serie Horticultura 5: 261-265. Consultado 19 mar 2014. Disponible en http://209.143.153.251/WAC4/W AC4_ p261.pdf

López-Herrera, CJ; Pérez- Jiménez, RM; Zea-Bonilla, T. 2003. Evaluación de diferentes fungicidas para el control de la Podredumbre Blanca del Aguacate (en línea). In $\mathrm{V}$ Congreso Mundial del Aguacate (5, Málaga, España) Acta. Málaga, España, Junta de Andalucía. Consultado 19 mar 2014. Disponible en http://www.avocadosource.com/W AC5/Papers /WAC5_p543.pdf

Manzur, M. 2011. Control de Sclerotium rolfsii con aislados nativos y comerciales de Trichoderma sp. in vitro. Tesis Ing. Agr. San Lorenzo, Paraguay, FCAUNA. $40 \mathrm{p}$.

Mendoza, R. 2000. Aislamiento selectivo y pretamizado en bioensayos de micoparásitos contra Rosellinia spp. (en línea). Tesis MSc. Turrialba, Costa Rica, Centro Agronómico Tropical de Investigación y Enseñanza. 107 p. Consultado 16 ene 2014. Disponible en http://orton.catie.ac.cr/repdoc/A0372E/A0372E.PDF 
Mendoza, R; Ten Hoopen, M; J Kass, D; Sánchez, V; Krauss. 2002. Evaluation of mycoparasites as biocontrol agents of Rosellinia root rot in cocoa (en línea). Sciencie direct 27(2): 210-227. Consultado 16 ene 2014. Disponible en http://www.science direct.com/science/article/pii/S104996 4403000148

Oliveira, ML; Melo, GL; Niella, AR; Silva, VR. 2008. Black root rot caused by Rosellinia pepo, a new disease of the clove tree in Brazil (en línea). Tropical plant pathology 33(2): 90-95. Consultado 16 ene 2014. Disponible em http://www.scielo.br/ scielo. php?pid=S198256762008000200002\&script=sci_artte $\mathrm{xt} \& \operatorname{tng}=\mathrm{pt}$

Orrego, A; Rodriguez, H; Grabowski, C; Franco, B. 2013. Comportamiento de aislados de Trichoderma spp. en cultivo pareado con Macrophomina phaseolina. In Orrego, A. Trichoderma spp. hongo biocontrolador de fitopatógenos. San Lorenzo, Paraguay, FCA-UNA/ INBIO. $129 \mathrm{p}$.

Papavizas, GC. 1985. Trichoderma and Gliocladium: biology, ecology, and potential for biocontrol (en línea). Annual review of Phytopathology 23(1): 23-54. Consultado 21 mar 2014. Disponible en http://www.annualreviews.org/doi/abs/10.1146/annure v.py.23.090185.000323?journalCode $=$ ph yto

Ruano-Rosa, D; López-Herrera, C. J. 2003. Control de la Podredumbre Blanca del Aguacate mediante métodos químicos y biológicos combinados (en línea). Córdoba, ES, Instituto de Agricultura Sostenible. Consultado 14 mar 2013. Disponible en http://worldavocadocongress2011 .com/userfiles/file/ Fernando\%20Pliego-Alfaro.pdf

Ruano-Rosa, D; Del Moral-Navarrete, L; López-Herrera. 2010. Selection of Trichoderma spp. isolates antagonistic to Rosellinia necatrix (en línea). Journal of Agricultural Research 8(4): 1084-1097. Consultado 14 mar 2013. Disponible en http://digital.csic.es /handle/1026 1/84178
Sharma, SK; Gupta, VK. 1985. Movement and persistence of fungicides in apple soils (en linea). Indian Phytopathology 38(1): 648-652. Consultado 17 mar 2013. Disponible en http://scholar.google.com .py/scholar?

Sir, EB; Perera, TC; Romero, AI; Hladki, AI. 2012. Novedades para el género Rosellinia (AscomycotaXylariaceae) en el Noroeste de la República Argentina (en línea). Boletín de la Sociedad Argentina de Botánica 47(3): 311-321. Consultado 12 mar 2014. Disponible en http://www.scielo.org.ar/scielo.ph p?pid $=\mathrm{S} 1851-23722012000200003 \&$ script $=$ sci_artte xt

Valencia, JC; Castro, BL. 2004. Estudio de algunos aspectos biológicos de aislamientos de Trichoderma sp. antagónicos a Rosellinia bunodes (en línea). Cenicafé 55(1): 16-28. Consultado 20 dic 2013. Disponible en http://biblioteca. cenicafe.org/handle/10 $778 / 222$

Villegas, C. 2005. Reconocimiento fitosanitario en cinco variedades cultivadas de macadamia (Macadamia integrifolia Maiden et Betche) en la zona cafetera colombiana (en línea). Manejo Integrado de Plagas y Agroecología 74(1): 69-76. Consultado 17 ago 2012. Disponible en orton.catie.ac.cr/repdoc

Villegas, C; Realpe, CE; Riaño, NM. 2006. Aislamiento y Caracterización Morfológica de Rosellinia pepo Pat. en plantas de Macadamia (en línea). Revista Facultad Nacional de Agronomía 59(2):3509-3526. Consultado 17 ago 2012. Disponible en http://www.scielo.org.co/s cielo.php?pid=S0304-28472006000200008\&script $=$ sci_arttext\&tlng=es 